\title{
The nSoft Autonomous Formulation Laboratory: SANS/SAXS/WAXS Liquid Handling for Industrial Formulation Discovery
}

\author{
P.A. Beaucage ${ }^{1,2}$, T.B. Martin ${ }^{1}$ \\ ${ }^{1}$ Materials Science \& Engineering Division, ${ }^{2}$ NIST Center for Neutron Research, National Institute of Standards and Technology, \\ Gaithersburg, MD 20899, United States \\ peter.beaucage@nist.gov
}

Complex liquid mixtures are the foundation of industrial products from personal care products to biotherapeutics to specialty chemicals. While small- and wide-angle reciprocal space methods (SANS, SAXS, WAXS) are workhorse techniques for characterizing model formulations, the large number of components (10-100) in many real products often prevents rational mapping between component fractions, structure, and product stability. To enable rational design of these materials, we must leverage theory, simulation, multimodal characterization and machine learning (ML) tools to greatly reduce the expense of exploring the stability boundaries of a particular, desirable phase. Applying ML tools to scattering experiments requires a platform capable of autonomously synthesizing and characterizing samples with varying composition and chemistry. While there are numerous examples of robots which perform specific user facility operations, these systems tend to be bespoke and non-adaptable to new tasks. We have developed a highly adaptable platform that can be programmed to autonomously prepare and characterize liquid-formulations using neutron and Xray scattering in addition to offline techniques such as optical imaging, UV/vis/NIR, viscometry, etc. Here we will highlight the design of the platform and our latest results in autonomous stability mapping of model formulations from personal care, biopharmaceutical, and alternative energy partner companies.

Keywords: autonomous experimentation, liquid handling, industrial formulations, soft matter

PAB thanks the NIST-NRC postdoctoral fellowship program for support. This work was supported by the member companies of the nSoft consortium at NIST (nist.gov/nsoft). 